\title{
Optimizing CPR Chest Compression in the EMS Environment
}

\author{
Samuel J. Stratton, MD, MPH
}

Emergency Medical Services (EMS) systems in many parts of the world transport patients in cardiac arrest while performing manual chest compressions. Other EMS systems have adopted strategies to avoid this procedure. One procedure is the use of mechanical chest compression devices when moving a patient, and another is termination of resuscitation efforts after thorough on-site Basic and Advanced Life Support resuscitation efforts when there is lack of return of spontaneous circulation (ROSC).

A recent study by Duval, et $\mathrm{al}^{1}$ has confirmed previous research on optimal chest compression rate and depth. In their paper, the authors report an optimal combination of 107 compressions per minute and compression depth of $4.7 \mathrm{~cm}$ during adult cardiopulmonary resuscitation (CPR). In the study, when CPR was performed within $20 \%$ of the optimal compression and depth combination, good neurologic outcome determined by a survival modified Rankin Score of less than or equal to three was reported to be $6.0 \%$ versus $4.3 \%$ (when compression rate and depth fell outside of $20 \%$ of the optimal range). Study findings were based on data obtained for the first five minutes after EMS personnel arrived and began performing CPR and did not extend to lengthier CPR efforts.

A question in EMS is the impact of unavoidable interruptions of manual chest compressions during CPR. Manual chest compression interruptions in the out-of-hospital environment occur when performing tasks such as defibrillation, intubation, transport of a patient onto a gurney (stretcher), loading into an ambulance, and offloading from the ambulance. Further challenging EMS providers is attempting to continue chest compressions while moving a patient already secured to a rolling gurney. Stairwells present a particular challenge for continuing manual chest compressions. In the EMS environment, it is illogical to assume that optimal and uninterrupted manual chest compressions during CPR occur beyond the site of the collapse of the patient.

Physiologically, it is recognizable that continuous chest compressions during $\mathrm{CPR}$ are required to maintain continuous cerebral and coronary blood flow and optimize potential for patient survival. Two strategies for minimizing or eliminating interruption of chest compressions by EMS responders are use of mechanical chest compression devices in place of manual compression and extending CPR and Advanced Life Support time on-site without movement of the patient until development of ROSC or further resuscitation efforts are considered futile. Both strategies limit interruption of chest compressions and maximize resuscitation cerebral and coronary blood flow, but also have limitations. In addition to eliminating interruption of chest compressions during CPR, these strategies have the benefit of decreasing the risk for injury or fatality of EMS personnel if transporting a cardiac arrest patient and performing manual chest compressions in a moving ambulance while unrestrained with seat and shoulder harnesses.

In their article, Duval, et al, suggest there may be benefit with the use of mechanical chest compression devices to ensure optimal

and continuous compression rate and depth. ${ }^{1}$ To optimize chest compression delivery and protect EMS staff during transport, a number of EMS systems in the world have adopted mechanical chest compression as the required standard for CPR chest compression. Mechanical chest compression technology was first introduced in the 1990s and the devices have been refined and improved over two decades. Initial case reports of chest, vascular, and solid organ injury presumed due to use of the devices have become uncommon. Interesting is that the early case reports of injury usually included both manual and mechanical chest compression. Superiority or inferiority of mechanical devices when compared to manual compression in controlled, static settings has not been shown. ${ }^{2}$ There is little practical EMS research comparing neurologic intact survival rates with use of mechanical compression versus on-going manual chest compression (an area that is ripe for future research).

A disadvantage for implementing mechanical chest compression into EMS systems is cost for purchase of devices, training, and maintenance. Cost associated with mechanical compression devices may be somewhat offset by decreased risk for injury of EMS personnel by eliminating the need for chest compressions while unrestrained during transport. Other than cost, an EMS standard of practice for CPR with mechanical chest compression is supported as a method to optimize chest compressions and improve EMS personnel safety during transport.

Another strategy to eliminate interruption of chest compression and improve EMS personnel safety during transport is to continue CPR and Advanced Life Support at the cardiac arrest site until ROSC, or until further resuscitation is futile and transport is unnecessary. On-going CPR with optimal chest compressions and maximum Advanced Life Support efforts at the scene of the cardiac arrest can be considered a limit for prolonging resuscitation if a patient does not have ROSC. A recent Swedish in-hospital study of patients with sustained ROSC shows that survival can occur with extension of resuscitation efforts out to 60 minutes. ${ }^{3}$ While there is limited information for survival related to time for out-of-hospital cardiac arrest, it is reasonable to develop field protocols that allow for discontinuation of resuscitation at the scene of a cardiac arrest if there is a lack of sustained ROSC following optimal CPR and exhaustion of Advanced Life Support actions. Disadvantages of this strategy are potential fatigue of rescuers who perform manual chest compressions, need to perform resuscitation in potential unstable settings (public or stressful home environments), local legal limitations for discontinuing EMS CPR, limited pharmaceuticals and resources available for field Advanced Life Support, and disposition of a deceased person's body if resuscitation is stopped. One difficult issue is the management of a patient who has intermittent episodes of ROSC. Some using the strategy of maximizing resuscitation on-site have added use of end-tidal carbon dioxide measurements to assess for a perfusing cardiac rhythm which would indicate the need for on-going 
resuscitation or transport. ${ }^{4}$ While difficult to address with a protocol or practice guideline approach, the strategy of discontinuation of resuscitation at the site of a cardiac arrest after all life support efforts appear futile addresses the EMS challenges of interrupted chest compressions during CPR and personnel safety during transport while performing manual chest compressions.

In summary, research has been published which shows optimal chest compression parameters for effective CPR. The concept of uninterrupted chest compressions during $\mathrm{CPR}$ to maintain cerebral and coronary blood flow is an accepted standard for resuscitation. But, in the EMS environment, there are unavoidable periods of interruption of manual chest compression if a patient is transported with CPR in progress. Two strategies to optimize chest compression during out-of-hospital resuscitation include either use of mechanical chest compression devices for field CPR and transport, or developing procedures that maximize field resuscitation at the site of the arrest until there is ROSC or a determination that further effort is futile. Both strategies have disadvantages, but to continue inevitable interruption of chest compressions in the out-of-hospital setting using manual chest compressions during patient movement and transport is illogical and ignores the basic physiologic basis for chest compressions during CPR.

\section{References}

1. Duval S, Pepe PE, Aufderheide TP, et al. Optimal combination of compression rate and depth during cardiopulmonary resuscitation for functionally favorable survival. JAMA Cardiol. 2019.

2. Brooks SC, Hassan N, Bighan BL, Morrison LJ. Mechanical versus manual chest compressions for cardiac arrest. Cochrane Database Syst Rev. 2014;27(2):CD002260.

3. Rohlin O, Taeri T, Netzereab S, Ullemark E, Djarv T. Duration of CPR and impact on 30-day survival after ROSC for inpatient cardiac arrest-a Swedish cohort study. Resuscitation. 2018;132:1-5.
4. Eckstein M, Hatch L, Malleck J, McClung C, Henderson SO. End-tidal CO2 as a predictor of survival in out-of-hospital cardiac arrest. Prehosp Disaster Med. 2011;26(3): 148-150.

doi:10.1017/S1049023X19004862 